\title{
Nuclear data evaluation of long-lived fission products: Microscopic vs. phenomenological optical potentials
}

\author{
Futoshi Minato $^{1, a}$, Osamu Iwamoto ${ }^{1}$, Kosho Minomo ${ }^{2}$, Kazuyuki Ogata ${ }^{2}$, Nobuyuki Iwamoto ${ }^{1}$, Satoshi Kunieda ${ }^{1}$, \\ and Naoya Furutachi ${ }^{1}$ \\ ${ }^{1}$ Nuclear Data Center, JAEA, Tokai, Japan \\ 2 Research Center for Nuclear Physics, Osaka Univ., Ibaraki, Japan
}

\begin{abstract}
Neutron-nucleus cross sections calculated by macroscopic potentials are compared with a microscopic one to study the performance for long-lived fission products. The macroscopic potentials show a good agreement with the microscopic one at higher energies, where neutron experimental data are scarce. Besides it, analyses of differential elastic cross sections at low energies also suggest that the macroscopic potentials are still effective and applicable enough for the long-lived fission products.
\end{abstract}

\section{Introduction}

Phenomenological optical potential is known to be able to describe the nuclear scattering process well. The advantage of the potential is that it can calculate the total and elastic scattering cross sections and the analyzing power successfully without considering complicated processes occuring in the target nuclei. Therefore, it is applied widely to the nuclear data evaluation of medium to heavy nuclei [1]. There are now many kinds of the optical potential parameterized both in local $^{1}$ and global forms.

Optical potentials are roughly divided into two kinds, that is, phenomenological and microscopic ones. The parameters for the former one are determined so as to reproduce existing experimental data of nucleon- or nucleus-nucleus reactions. In particular, the global optical potential parameters, which have a phenomenological function of mass and energy, are determined by making use of experimental data of arbitrarily chosen nuclei ranging from light to heavy mass region. From the fact that the obtained parameter sets usually have moderate mass and energy dependences, one can also obtain a reasonable result of cross sections for other nuclei not included in the parameterization process, using the same potential. However, it will be not necessarily reliable for unmeasured nuclei apart from the stability line such as neutronrich nuclei including fission products. The microscopic approach, on the other hand, constructs optical potentials starting from a two-body nucleon-nucleon interaction considering the medium effect. Since it does not require any arbitrary function, the microscopic optical potential is

\footnotetext{
a e-mail: minato.futoshi@jaea.go.jp

${ }^{1}$ The word "local" here indicates optical potential parameterized for a certain nucleus or a certain energy.
}

in principle able to provide reliable optical potentials for all the nuclei in the nuclear chart.

Minomo et al. have recently studied microscopic optical potentials [2] and obtained a result that proton elastic scattering from ${ }^{90} \mathrm{Zr}$ is described well with the potential. It was also confirmed that the optical potential can reproduce experimentally measured elastic scattering cross sections from light to heavy nuclei successfully if the density distributions of target nuclei are correctly given [3]. In addition, Toyokawa et al. also investigated elastic scattering cross sections of several nuclei with polarized protons with this potential [4]. The optical potentials are derived from the nucleon-nucleus folding model with the g-matrix of the Melbourne interaction [5] constructed from the Bonn-B NN interaction [6]. However, the Minomo potential is not perfect either; it cannot be applied to scattering processes at lower energies since it takes into account only the so-called knock-on exchange, and assumes some approximations to localize the exchange part of the folding potential [2].

Although microscopic optical potentials will be used widely for nuclear data evaluations in the future, phenomenological one is still important and useful. However, it would be more convincing if one would check if phenomenological potential works for unstable nuclei, especially for long-lived fission products, which have attracted many interests due to its nuclear transmutation in some way. We therefore assess the performance of the phenomenological optical potential by comparing them with the Minomo potential. We mainly pay attention to neutron-nucleus cross sections from $E_{n} \sim 65 \mathrm{MeV}$, where the approximations used in the Minomo potential appear to work well [2], to $200 \mathrm{MeV}$. Such an energy region is our interest because the largest ambiguity will arise from there since neutron cross section data at high energies are scarcer than at low energies in most nuclei. The cross sections below $E_{n}=65 \mathrm{MeV}$ are also discussed because we may obtain a hint to extend the microscopic approach to the lower energies. 

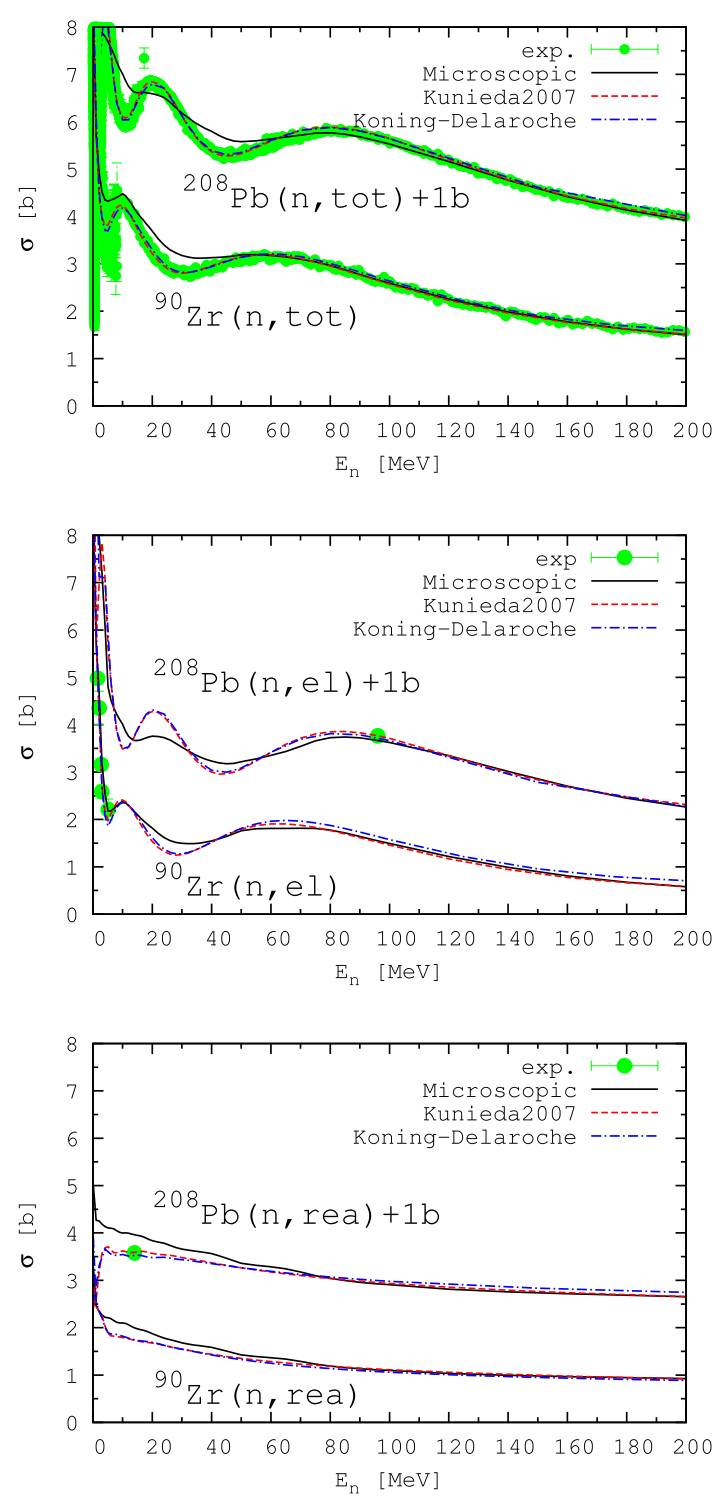

Figure 1. Total (the top panel), elastic (middle), and reaction (bottom) cross sections as a function of neutron energy for ${ }^{90} \mathrm{Zr}$ and ${ }^{208} \mathrm{~Pb}$ are shown. The solid, dashed, and dotted-dashed lines indicate the results of Minomo, Kunieda2007, and KoningDelaroche potentials, respectively. The experimental data are taken from the EXFOR database [9].

\section{Comparison between phenomenological and microscopic optical potentials}

We compare cross sections and differential cross sections of neutron-nucleus reactions calculated by the Minomo potentials with those of two widely used phenomenological ones, the first being the KoningDelaroche global optical potential [7] and the other being the Kunieda potential (Kunieda2007) [8]. The KoningDelaroche potential parameters are tuned by available experimental data of about 70 nuclei and those of the Kunieda2007 about 40 nuclides including those with natural abundance ratio, in which any unstable nuclei are not included.

We begin with comparing results of stable nuclei, ${ }^{90} \mathrm{Zr}$ and ${ }^{208} \mathrm{~Pb}$. Figure 1 shows the total, elastic, and
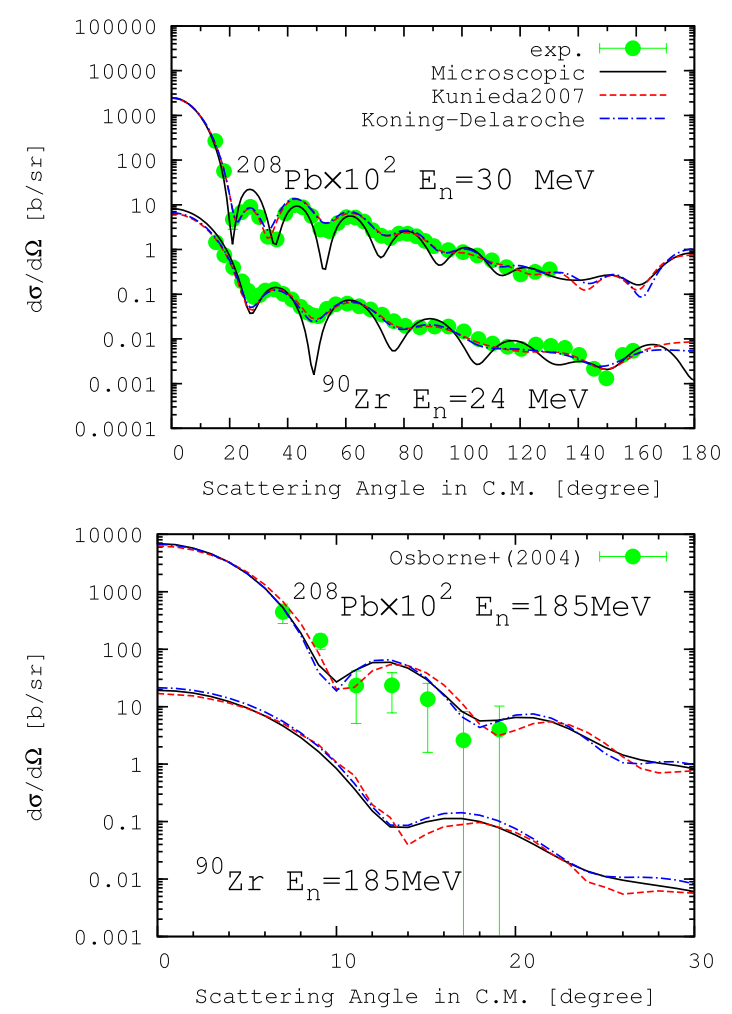

Figure 2. Differential elastic scattering cross sections of ${ }^{90} \mathrm{Zr}$ and ${ }^{208} \mathrm{~Pb}$ are shown. The top and bottom panels illustrate the results of $E_{n}=24 \mathrm{MeV}$ for ${ }^{90} \mathrm{Zr}$ and $30 \mathrm{MeV}$ for ${ }^{208} \mathrm{~Pb}$, and $E_{n}=185 \mathrm{MeV}$, respectively. The solid, dashed, and dotted-dashed lines indicate the results of Minomo, $\mathrm{Ku}$ nieda2007, and Koning-Delaroche potentials, respectively. The experimental data are taken from the EXFOR database [9] and Ref. [10].

reaction cross sections for ${ }^{90} \mathrm{Zr}$ and ${ }^{208} \mathrm{~Pb}$ calculated with the Minomo and the phenomenological potentials. Two phenomenological potentials reproduce experimental data of total cross section well for both nuclei. The same result is obtained in the elastic cross section. The Minomo potential also reproduces experimental data of total and elastic scattering cross sections and shows a close curve to two phenomenological calculations above $60 \mathrm{MeV}$ for ${ }^{90} \mathrm{Zr}$ and $80 \mathrm{MeV}$ for ${ }^{208} \mathrm{~Pb}$. In particular, the results of the Kunieda2007 potential are slightly closer to those of the Minomo potential than those of the Koning-Delaroche one.

Figure 2 shows the differential elastic cross sections of ${ }^{90} \mathrm{Zr}$ and ${ }^{208} \mathrm{~Pb}$ at lower energy (the top panel) and higher energy (bottom). As seen in the top panel, two phenomenological optical potentials show a good agreement with experimental data. Though the application of microscopic calculation to incident neutron energy less than $65 \mathrm{MeV}$ is not guaranteed, the result also reproduces the experimental data roughly, but the diffraction structure is not good. The bottom panel shows the results at $E_{n}=$ $185 \mathrm{MeV}$. The experimental data of ${ }^{208} \mathrm{~Pb}$ are reproduced reasonably well by all potentials. The difference between the Minomo potential and the phenomenological ones is smaller than the lower energy case. In particular, the Koning-Delaroche potential result is closer to the Minomo potential one than the Kunieda2007 one. 

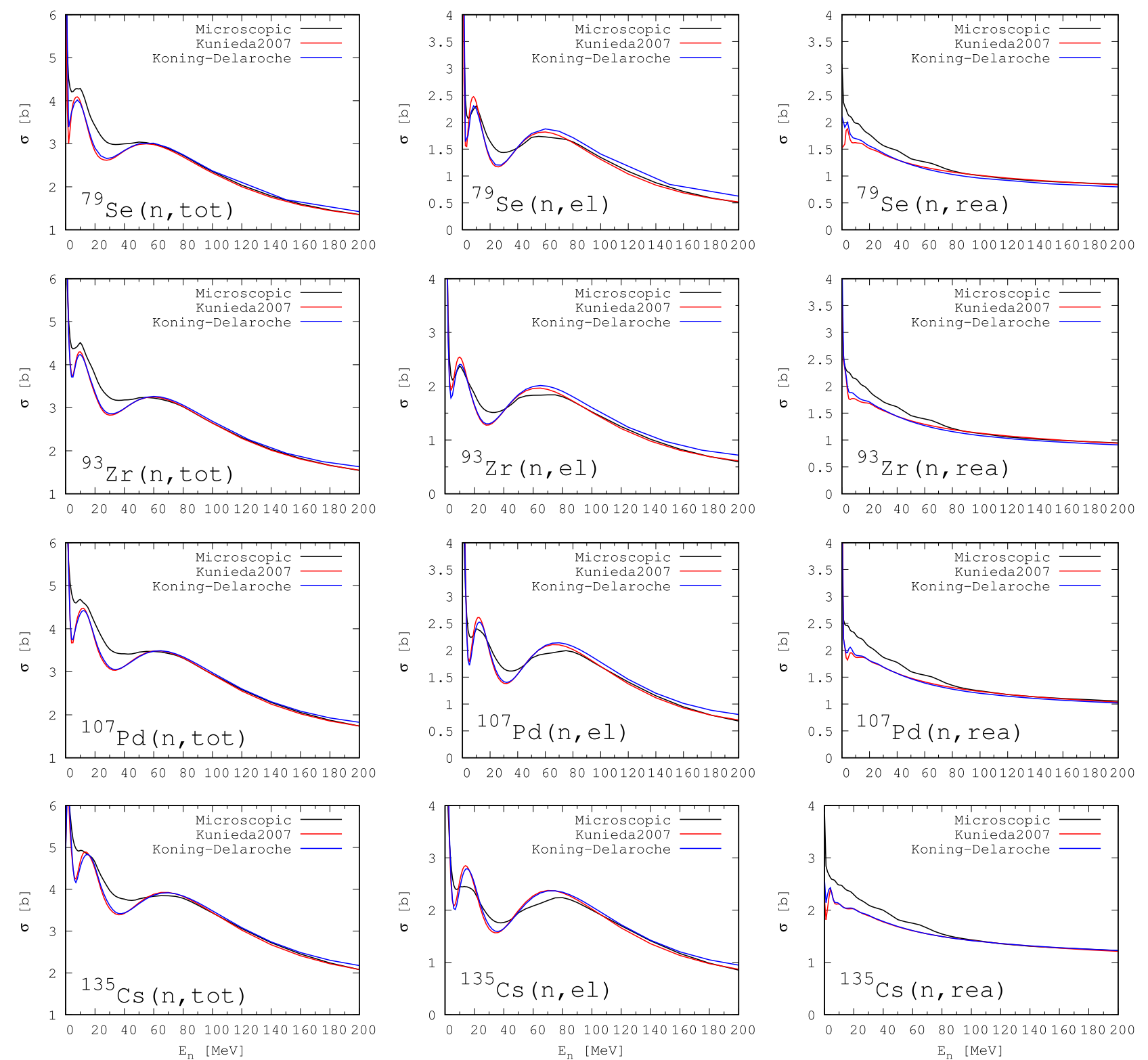

Figure 3. Total (the left panel), elastic (middle), and reaction (right) cross sections as a function of neutron energy for long-lived fission products, ${ }^{79} \mathrm{Se},{ }^{93} \mathrm{Zr},{ }^{107} \mathrm{Pd}$, and ${ }^{135} \mathrm{Cs}$, are shown. The solid, dashed, and dotted-dashed lines indicate the results of Minomo, Kunieda2007, and Koning-Delaroche potentials, respectively.

Figure 3 illustrates the total, elastic, and reaction cross sections for long-lived fission products, ${ }^{79} \mathrm{Se},{ }^{93} \mathrm{Zr}$, ${ }^{107} \mathrm{Pd}$, and ${ }^{135} \mathrm{Cs}$. Similar to the stable nuclei, two phenomenological optical potentials give almost the same results, and the Minomo potential also show a similar curve from $E_{n}=60-80 \mathrm{MeV}$. However, the Kunieda2007 potential result is slightly closer to the Minomo potential result than the Koning-Delaroche one. Figure 4 shows the differential elastic cross sections for the long-lived fission products of $E_{n}=80,160 \mathrm{MeV}$. The diffraction patterns are almost the same for all the potential. The same result is also obtained for the middle-lived fission products ${ }^{90} \mathrm{Sr}$ and ${ }^{137} \mathrm{Cs}$.

\section{Conclusion}

We compared the results of the cross sections calculated by the phenomenological and the microscopic optical potentials. In particular, we paid attention to neutronnucleus cross sections at high energies, where the approximations used in the Minomo potential appear to work well. As mentioned in Sect. 1, such an energy region is our interest because the largest ambiguity arises from there due to the shortage of experimental data.

We obtained the result that the cross sections calculated by the phenomenological potentials are close to those of the microscopic one above $60-80 \mathrm{MeV}$, in which the Minomo potential can be applicable, for the long-lived fission products as well as the stable nuclei. This is one of the implications that the Koning-Delaroche and the Kunieda potentials are still valid for those nuclei at least at high energies. At lower energies, two phenomenological potentials used in this study show almost the same result. In addition, the long-lived fission products studied in this work are not far from the stable nuclei, so that the global parameters are still effective, and therefore we consider 

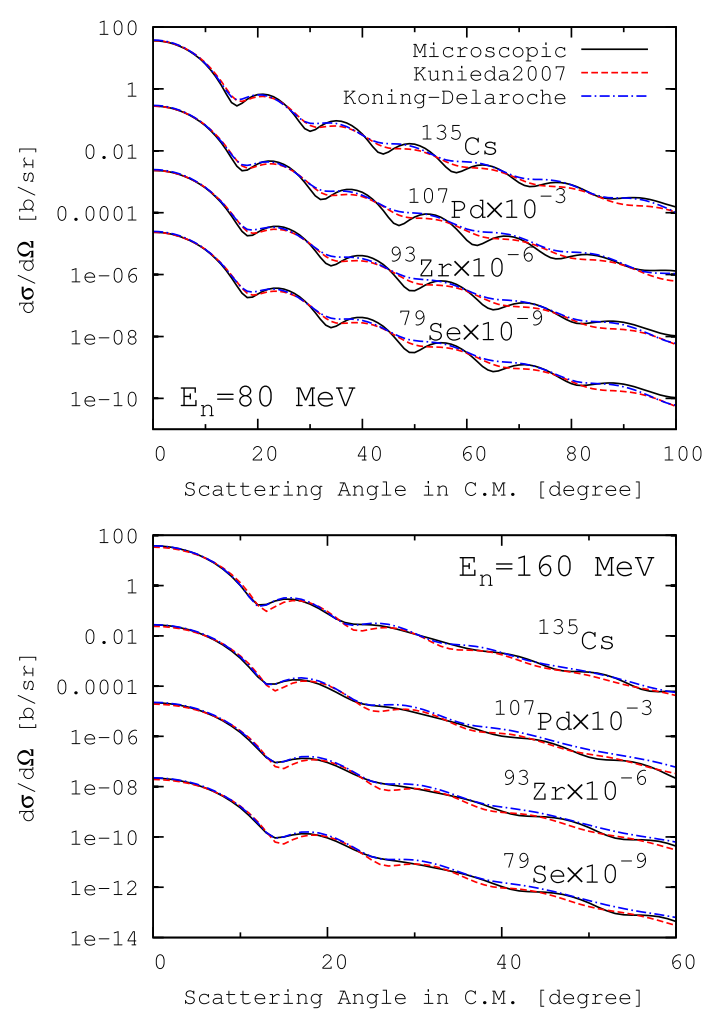

Figure 4. Differential elastic scattering cross sections for ${ }^{79} \mathrm{Se}$, ${ }^{93} \mathrm{Zr},{ }^{107} \mathrm{Pd}$, and ${ }^{135} \mathrm{Cs}$ at neutron energies $E_{n}=80$ (the top panel) and $160 \mathrm{MeV}$ (bottom).

that the phenomenological potentials are applicable for those nuclei.
We did not discuss the deformation effect in this work, which becomes important at low energies. The effect should be taken into account in the paractical evaluations including low energy regions of the long-lived fission products.

This work was funded by ImPACT Program of Council of Science, Technology and Innovation (Cabinet Office, Government of Japan).

\section{References}

[1] K. Shibata, et al., J. Nucl. Sci. Technol. 48, 1 (2011)

[2] K. Minomo, K. Ogata, M. Kohno, Y.R. Shimizu, and M. Yahiro, J. Phys. G: Nucl. Part. Phys. 37, 085011 (2010)

[3] K. Minomo, private communication (2015)

[4] M. Toyokawa, K. Minomo, and M. Yahiro, Phys. Rev. C 88, 054602 (2013)

[5] K. Amos, P.J. Dortmans, H.V. von Geramb, S. Karataglidis, and J. Raynal, Adv. Nucl. Phys. (Plenum Publishers, New York, 2000) 25, 275

[6] R. Machleidt, K. Holinde, and Ch. Elster, Phys. Rep. 149, 1 (1987)

[7] A.J. Koning and J.P. Delaroche, Nucl. Phys. A713, $231(2003)$

[8] S. Kunieda, S. Chiba, K. Shibata, A. Ichihara, and E.Sh. Sukhovitskiĩ, J. Nucl. Sci. Technol. 44, 838 (2007)

[9] N. Otuka, et al., Nucl. Data Sheets 120, 272 (2014); http://www.nndc.bnl.gov/

[10] J.H. Osborne, F.P. Brady, J.L. Romero, et al., Phys. Rev. C 70, 054613 (2004) 